\title{
Temperature Dependence of the Diffusion Coefficient of Polystyrene Latex Spheres
}

\author{
ALINA C. FERNANDEZ and GEORGE D. J. PHILLIES, Department of \\ Chemistry, The University of Michigan, Ann Arbor, Michigan 48109
}

It has long been assumed that the effect of temperature on the diffusion coefficient $D$, the sedimentation coefficient $s$, and other hydrodynamic parameters is correctly described by Walden's rule

$$
D_{1} \eta_{1} / T_{1}=D_{2} \eta_{2} / T_{2}
$$

and its extensions, i.e., hydrodynamic properties of dilute solutions of macromolecules scale linearly with temperature and antilinearly with solvent viscosity. The accuracy of this rule was extensively studied by Longsworth, ${ }^{1}$ who used a Rayleigh interference technique to measure the diffusion coefficient of materials with molecular weights of 20-68,000. While one cannot show the universal applicability of a rule, over the temperature range of $1-37^{\circ} \mathrm{C}$, the diffusion coefficients of the macromolecular solutes studied in Ref. 1 were found to deviate from Eq. (1) by only $\pm 4 \%$.

Recently, Crossley et al. ${ }^{2}$ used quasielastic light scattering (QELS) to measure the diffusion coefficient of polystyrene latex and low-density lipoprotein over the temperature range $25-50^{\circ} \mathrm{C}$, finding large deviations $(-0.6 \% / \mathrm{K})$ of $D$ from Walden's rule. These workers also obtained the drag coefficient $f$ of low-density lipoprotein by ultracentrifugation, finding a similar anomaly in the temperature dependence of $f$. The paper of Crossley et al. ${ }^{2}$ thus joins a series of papers ${ }^{3}$ suggesting that QELS may not always measure the conventional mutual diffusion coefficient.

It is somewhat difficult to understand how such a large effect could have been overlooked in earlier work. The anomaly found by Crossley et al. ${ }^{2}$ is smallest near room temperature, measured and calculated values for $D$ being in near agreement at $25^{\circ} \mathrm{C}$. Since there have been relatively few studies of the temperature dependence of QELS spectra of macromolecule solutions, ${ }^{4,5}$ it is conceivable that the agreement between light-scattering spectroscopy and other methods is in part a coincidence that unfortunately occurs at the standard measurement temperature. Alternately, polystyrene latex spheres might be subject to a hitherto unnoticed change in diameter with temperature. Since these spheres are a calibration standard in light-scattering spectroscopy, an unnoticed dependence of their radius on $T$ could lead to systematic errors in other experiments.

In order to test these hypotheses, we made extensive measurements on the diffusion of $0.038-\mu \mathrm{m}$ carboxylate-modified polystyrene latex spheres (Dow Diagnostics) over the temperature range $0-50^{\circ} \mathrm{C}$. The spheres were suspended in 14 $\mathrm{M} \Omega$ deionized water at a concentration of $\sim 5 \times 10^{-4}$ by volume. At this concentration, multiple scattering and sphere-sphere forces are negligible. Our instrumentation and data-analysis techniques are unchanged from our earlier work. ${ }^{4,5}$

The observed spectra, $S(k, t)$, were fit to a cumulant expansion. ${ }^{6}$ The best fit was usually obtained with a second-or, rarely, a third-order fit. The variance $V$ 
$=100 \sqrt{ } K_{2} / K_{1}$, which is a reliable test for moderate levels of sample polydispersity, ranged from 20 to $35 \%$ in most spectra. $V$ was not correlated with the temperature or age of the sample. Measurements on a given sample at different temperatures were made in series proceeding away from $25^{\circ} \mathrm{C}$; on restoring a sample to $25^{\circ} \mathrm{C}$, the measured diffusion coefficient returned to its original value at $25^{\circ} \mathrm{C}$. This would not be expected if cycling the sample in temperature over a $4-8$-h period caused an irreversible aggregation of the polystyrene spheres.

Our experimental results are presented in Fig. 1, which plots the measured mutual diffusion coefficient $D$ of the polystyrene latex spheres as a function of $T / \eta$. The viscosity of pure water was obtained from standard tables. ${ }^{7}$ Each dot represents an average over three to five independent spectra; the original spectra agreed with each other to within $\pm 1 \%$. The solid line is a least-mean-squares fit to the data; the data fit the equation

$$
D=0.00359 T / \eta-0.0221
$$

with the units being $\left(D, 10^{-7} \mathrm{~cm}^{2} / \mathrm{s} ; T / \eta, \mathrm{K} / \mathrm{cp}\right.$. $)$ This corresponds to a sphere radius of $204 \AA$, slightly larger than the manufacturer's nominal $190 \AA$. Disagreements concerning this size and sign are to be expected between QELS and other physical techniques.

There is no apparent systematic deviation of the data from the straight line. To within experimental error (roughly $\pm 0.02 \times 10^{-7} \mathrm{~cm}^{2} / \mathrm{s}$ ), the intercept is zero. The spheres diffuse as though their apparent hydrodynamic radius is independent of temperature:

$$
r_{0}=K_{B} T / 6 \pi \eta D
$$

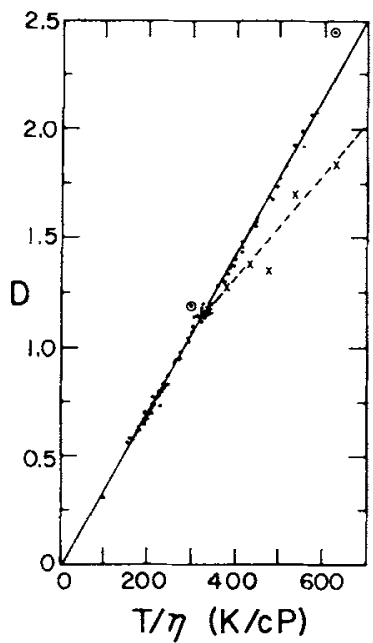

Fig. 1. Mutual diffusion coefficient of $0.038-\mu \mathrm{m}$ polystyrene latex spheres in pure water, plotted as a function of temperature over viscosity. The straight line is from a least-squares fit to Eq. (2). There are no apparent systematic differences between the data and the line. The $T / \eta=0$ intercept is not significantly different from zero. The crosses and dashed line indicate the temperature dependence of $D$ observed by Crossley et al. (Ref. 2), as normalized to agree with the diffusion coefficient of our polystyrene spheres at $25^{\circ} \mathrm{C}$. The two circled data points of ours were not included in the linear fit for Eq. (2). 
For purposes of comparison, the temperature dependence of $D$ found by Crossley et al., ${ }^{2}$ on their polystyrene sphere preparations under similar conditions, normalized to agree with our results at $25^{\circ} \mathrm{C}$ is indicated (crosses, dashed line). The disagreement between the two sets of data is outside the dispersion in our measurements.

We have previously reported $d^{4,5}$ on the use of optical probes in the study of diffusion in complex solvents, optical labeling being accomplished by a proper choice of the indexes of refraction of the probe and the solvent components. Studies have been made of the diffusion of $0.038-\mu \mathrm{m}$ polystyrene spheres in water:polyacrylic acid, ${ }^{5} 0.091-\mu \mathrm{m}$ polystyrene spheres in water:glycerol and water:sorbitol, ${ }^{4}$ and bovine serum albumin in water: $0.15 \mathrm{M} \mathrm{NaCl}$ :glycerol. ${ }^{4}$ With a fixed composition for these multicomponent solvents, the apparent hydrodynamic radius of each probe species is independent of temperature over the range $5-50^{\circ} \mathrm{C}$. For probes suspended in small-molecule solvents (water:glycerol, water:sorbitol), the hydrodynamic radius is also independent of the solvent composition. In contrast, the apparent hydrodynamic radius of a macroparticulate probe in a polymer-containing solvent does depend on the polymer concentration, but the temperature dependence of $D$ is still given by Walden's rule. The diffusion of probe particles in mixed polymeric systems may be significant for diffusion in living cells but is not a conventional hydrodynamic study.

In conclusion, we have repeated the light-scattering spectroscopic measurements of Crossley et al.,2 who reported an anomalously large temperature coefficient for $D$. We did not see such an effect. Other recent studies on polystyrene spheres in mixed solvents, which also failed to reveal a large temperature coefficient for $D$, are noted. We did not attempt to duplicate either Crossley et al.'s work on protein solutions or on the ultracentrifuge. We cannot suggest an experimental artifact that could create the discrepancy. The fear that polystyrene latex spheres are an unreliable size standard above $25^{\circ} \mathrm{C}$ appears unfounded.

This work was supported in part by National Science Foundation grant CHE 79-20389.

\section{References}

1. Longsworth, L. G. (1954) J. Phys, Chem. 58, 770-773.

2. Crossley, J. M., Spraggs, S. P., Creeth, J. M., Noble, N. \& Slack, J. (1982) Biopolymers $21,233-248$.

3. Minton, A. P. \& Ross, P. D. (1978) J. Phys. Chem. 82, 1934-1938.

4. Phillies, G. D. J. (1982) J. Phys. Chem. 85, 2838-2843.

5. Lin, T-H. \& Phillies, G. D. J. (1982) J. Phys. Chem. 86, 4073-4077.

6. Koppel, D. E. (1972) J. Chem. Phys. 57, 4814-4820.

7. Washburn, E. W., Ed. (1926) International Critical Tables, McGraw-Hill New York.

Received August 31, 1982

Accepted October 29, 1982 